\title{
Study of Variable Structure Control for Robot Based on Filter
}

\author{
Caihong Zhu \\ Suzhou Vocational University, Suzhou, Jiangsu, China \\ zhuzhang_2000@163.com
}

Keywords: sliding-mode; variable structure control; robot control.

\begin{abstract}
The sliding-mode variable structure control strategy is employed to control the Robot system. Firstly, the research work is emphasized on the description of the sliding mode surface and sliding mode condition. Then, the stability of the control system is ensured by Lyapunov method. Finally, simulation result is given to prove the validity of the variable structure controller.
\end{abstract}

\section{Introduction}

Since the formation of variable structure control theory to the short decades, now has become an important branch of control theory. Variable structure control method has been widely used in robot control system. The basic idea of variable structure control is that how to find a hyperplane to make all state trajectory converge to zero of the hyperplane in the state space of error system. Then, the state of the error system can reach the plane and go along the sliding plane to the origin through the continuous switching structure of the controller. Good robustness is an important advantage of variable structure control, which is mainly manifested in the invariance of the sliding mode motion equations for disturbance. As long as the selection of the large enough control signal is correct, under any disturbance, no matter from which an initial state, the state trajectory can reach the sliding mode in a reliable way. It is based on the advantages of sliding mode variable structure control method, which can be well used in robot control.

\section{Robot Dynamic Equation}

The robot dynamic equation based on Lagrange kinematics is:

$M(q) \ddot{q}+C(q, \dot{q}) \dot{q}+G(q)=\tau$

In the equation, $q$ and $\dot{q}$ are respectively the position and velocity of each joint of the robot, $\tau$ is driving moment vector.

$M, C, G$, respectively, is determined by the concrete structure of the robot of $n \times n, n \times n$ and $n \times 1$ order function matrix, $y$ is $n \times 1$ order moment vector.

A robot is a highly nonlinear system, its dynamics has some important features, which has considerable effect to reduce the calculation for measuring tool. The main conditions have the following several conditions:

(1) The positive definite symmetry: for any matrix $q, M(q)$ is positive definite symmetric.

(2) Boundedness: matrix function $M(q)$ and $C(q, \dot{q})$ for all matrix, $q$ and $\dot{q}$ are uniformly bounded.

(3) Oblique symmetry: matrix function $\dot{M}(q)-2 C(q, \dot{q})$ for any $q, \dot{q}$ is skew symmetric. For an arbitrary vector $\xi$, there is:

$$
\xi^{T}(\dot{M}(q)-2 C(q, \dot{q})) \xi=0
$$

\section{Sliding mode controller design and Lyapunov stability analysis}

The basic steps include two relatively independent parts when the sliding mode variable structure controller is designed:

(1) The design of the switch function $s(x)$, which make it sure that the sliding mode is asymptotically stable and have good dynamic quality; 
(2) The design of sliding mode control law $u^{ \pm}(x)$, which make conditions are met, thus form sliding mode area on the surface of the switch.

Once the switching function and sliding mode control law is got, the sliding mode control system can be fully established.

Using the low-pass filter in sliding mode controller output, can effectively filter the high frequency chattering control signal. A robotic sliding mode control system based on filter structure is shown in figure 1 .

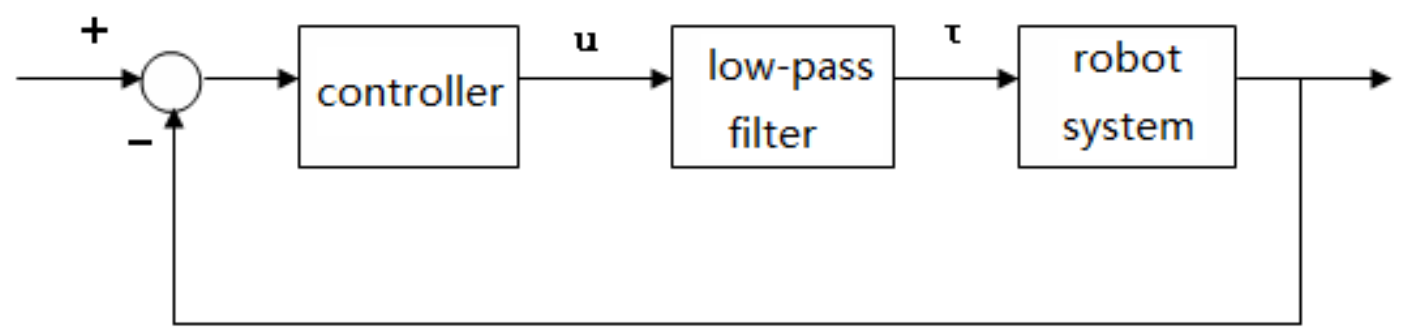

Fig. 1

Using low pass filter as follows:

$$
Q(s)=\frac{\lambda_{i}}{s_{i}+\lambda_{i}}
$$

In the equation $\lambda_{i}>0$

It can be got from available figure 1:

$\dot{\tau}+\Lambda \tau=\Lambda u$

Among the equation: $\Lambda=\operatorname{diag}\left(\lambda_{1}, \lambda_{2}, \Lambda, \lambda_{n}\right), \quad \lambda_{i}>0, i=1,2, \Lambda, n, n$ is the number of the input control signal

Will Eq. 1 into Eq. 4

$$
M \ddot{q}+\dot{M} \ddot{q}+C \ddot{q}+\dot{C} \dot{q}+\dot{G}+\Lambda M \ddot{q}+\Lambda C \dot{q}+\Lambda G=\Lambda u
$$

Setting up instructions $q_{d}(t)$ for the ideal location, the tracking error is following:

$$
e(t)=q(t)-q_{d}(t)
$$

The sliding mode function can be designed as following:

$$
s(t)=\ddot{e}+\Lambda_{1} \dot{e}+\Lambda_{2} e
$$

In the equation

$\Lambda_{i}=\operatorname{diag}\left(\Lambda_{i 1}, \Lambda_{i 2}, \Lambda, \Lambda_{i n}\right), \Lambda_{i j}>0, i=1,2, j=1,2, \Lambda, n$.

Stability is a basic structure characteristic of the system and the stability problem is an important hot topic in the field of system control theory. More than a century ago, Russia's mechanics A.M.Lyapunov published the thesis of the general problem of motion on the stability in 1892, the general theory of all movement stability is put forward firstly. This theory described by ordinary differential equations of dynamics system stability analysis is divided into two different essentially kinds of methods, now known as the Lyapunov's first method and the second method. Lyapunov method is applicable to linear systems and nonlinear systems, the time-varying system and time invariant system, continuous time systems and discrete time systems at the same time.

The Lyapunov's first method also known as the Lyapunov indirect method, belonging to the stability analysis method of a small scale. The discussions of the stability in the classical control theory is based on the basis of Lyapunov indirect method.

Lyapunov second method also known as the Lyapunov direct method, the method is presented internal stability directly based on the system structure. The second method directly used in the nonlinear systems, established the corresponding conclusion of judgment on the stability system, and is based on the introduction of Lyapunov function and the analysis with generalized energy attribute set number of Lyapunov function derivative. 
Since the Lyapunov second method was introduced into system control theory, it shows its importance in theory and application, and become o the main tools in the study on the stability of the modern system control theory. The stability analysis of the system based on Lyapunov second method is followed.

Define the function as

$V=\frac{1}{2} s^{T} M s$

Then

$\dot{V}=\frac{1}{2}\left(\dot{s}^{T} M s+s^{T} \dot{M} s+s^{T} M \dot{s}\right)$

Combining with several main characteristics of robot system, there is available:

$\dot{V}=s^{T} M \dot{s}+s^{T} C s=s^{T}(M \dot{s}+C s)=s^{T}\left[M\left(\dddot{q}-\dddot{q}_{d}+\Lambda_{1} \ddot{e}+\Lambda_{2} \dot{e}\right)+C\left(\ddot{e}+\Lambda_{1} \dot{e}+\Lambda_{2} e\right)\right]$

It can be obtained from the Eq. 5 :

$$
\begin{aligned}
M \ddot{q}=\Lambda u-\dot{M} \ddot{q}-\Lambda M \ddot{q}-C \ddot{q}-\dot{C} \dot{q}-\Lambda C \dot{q}-\dot{G}-\Lambda G \\
=\Lambda u-(\dot{M}+\Lambda M) \ddot{q}-(\dot{C}+\Lambda C) \dot{q}-(\dot{G}+\Lambda G)-C \ddot{q}
\end{aligned}
$$

Then:

$\dot{V}=s^{T}\left[\Lambda u-(\dot{M}+\Lambda M) \ddot{q}-(\dot{C}+\Lambda C) \dot{q}-(\dot{G}+\Lambda G)-C \ddot{q}+M\left(-\dddot{q}_{d}+\Lambda_{1} \ddot{e}+\Lambda_{2} \dot{e}\right)+C\left(\ddot{e}+\Lambda_{1} \dot{e}+\Lambda_{2} e\right)\right] \mathrm{a}$ nd, last

$I^{\&}=s^{T}(\Lambda u+H)$

In the equation:

$H=M\left(\Lambda_{1} \ddot{e}+\Lambda_{2} \dot{e}-\dddot{q}_{d}\right)+C\left(\Lambda_{1} \dot{e}+\Lambda_{2} e-\ddot{q}_{d}\right)-(\dot{M}+\Lambda M) \ddot{q}-(\dot{C}+\Lambda C) \dot{q}-(\dot{G}+\Lambda G)$

According to the structure type of the robot in the reference [3], $H$ can be written as

$H=\Gamma\left(t, q, \dot{q}, \ddot{q}, q_{d}, \dot{q}_{d}, \ddot{q}_{d} \dddot{q}_{d}\right) \varphi$

Then $\quad V^{2}=s^{T}(\Lambda u+\Gamma \phi)$

Designing the sliding mode control law for:

$u=-\Lambda^{-1}\left[\Gamma \phi_{0}+(\bar{\Gamma} \xi) \operatorname{sgn}(s)+\Psi \operatorname{sgn}(s)\right]$

In the equation: $\Gamma \phi_{0}=H_{0}, H_{0}$ is the accurate object of $H, \bar{\Gamma}_{i j}=\left|\Gamma_{i j}\right|, \Psi=\operatorname{diag}\left(\Psi_{1}, \Psi_{2}, \Lambda, \Psi_{n}\right)$, $\Psi_{i}>0, i=1,2, \Lambda, n, \operatorname{sgn}(s)=\left[\operatorname{sgn}\left(s_{1}\right) \operatorname{sgn}\left(s_{2}\right) \Lambda \operatorname{sgn}\left(s_{n}\right)\right]$. Then

$$
\begin{aligned}
& I \&=s^{T}\left[\Gamma\left(\phi-\phi_{0}\right)-(\bar{\Gamma} \xi) \operatorname{sgn}(s)-\Psi \operatorname{sgn}(s)\right]=s^{T}[\Gamma \bar{\phi}-(\bar{\Gamma} \xi) \operatorname{sgn}(s)-\Psi \operatorname{sgn}(s)] \\
& =\sum_{i=1}^{n}\left\{s_{i}(\Gamma \bar{\phi})_{i}-\left|s_{i}\right|(\bar{\Gamma} \xi)_{i}\right\}-s^{T} \Psi \operatorname{sgn}(s) \leq-s^{T} \Psi \operatorname{sgn}(s)=-\sum_{i=1}^{n}\left(\Psi_{i}\left|s_{i}\right|\right) \leq 0
\end{aligned}
$$

We assume $\eta=\bar{\Gamma} \xi+\Psi$, the control law can write to again:

$$
u=-\Lambda^{-1}\left[\Gamma \phi_{0}+\eta \operatorname{sgn}(s)\right]
$$

\section{Matlab Simulation and Analysis}

The dynamic equation of the single input single output of the single joint robot is following:

$M(q) \ddot{q}+C(q, \dot{q}) \dot{q}+G(q)+d(t)=\tau$

$M, C, G$, respectively, is determined by the specific structure of the robot, $d(t)$ is not sure part of the object modeling. In order to simulate, we assume that $M(q)=0.1+0.05 \cos (q)$, $C(q, \dot{q})=0.02 \sin (q), G(q)=0.1 \cos (q), d(t)=\sin (2 \pi t)$, and we also assume that $q_{d}=\sin (\pi t)$, the initial state of system is $\left[\begin{array}{ll}0.5 & 0\end{array}\right]^{T}$, there is one control input signal, which is $n=1$, we assume that $\Lambda_{1}=\lambda_{1}=5, \Lambda_{2}=\lambda_{2}=50, \Lambda=\lambda=20$, and control parameter is $\eta=1.5$.

Then $\quad u=-\Lambda^{-1}\left[H_{0}+\eta \operatorname{sgn}(s)\right]$ 
In the equation:

$$
H_{0}=M\left(\Lambda_{1} \ddot{e}+\Lambda_{2} \dot{e}-\dddot{q}_{d}\right)+C\left(\Lambda_{1} \dot{e}+\Lambda_{2} e-\ddot{q}_{d}\right)-(\dot{M}+\Lambda M) \ddot{q}-(\dot{C}+\Lambda C) \dot{q}-(\dot{G}+\Lambda G)
$$

The method of Variable-step, ode45(Dormand-Prince) is used in the simulation. The simulation results is shown in following figures.

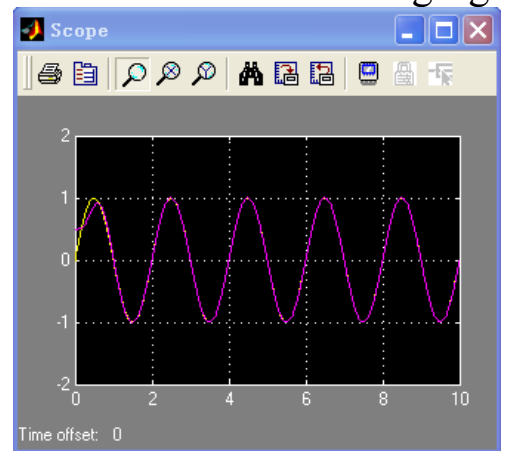

Fig. 2 location tracking

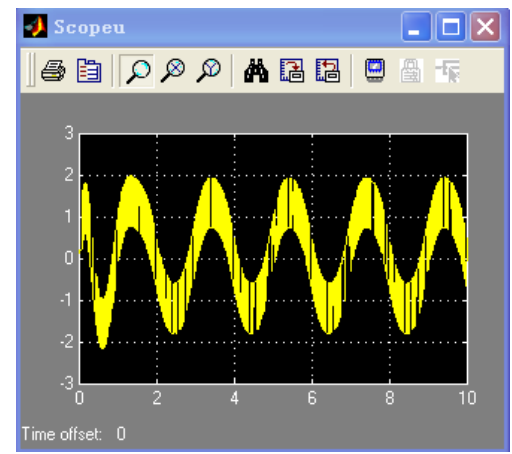

Fig. 3 input signal $u$

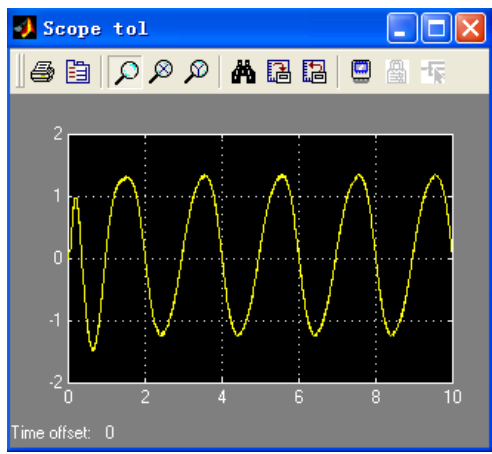

Fig. 4 input signal $\tau$

It is visible shown that, using low pass filter can significantly weaken chattering, and the simulation results namely verified the feasibility and effectiveness of the proposed algorithm.

\section{Conclusion}

Sliding mode variable structure control has a characteristic of good robustness, simple algorithm and easy to implement online waiting of the nonlinear uncertain factors, the study on its application also received considerable attention in recent years, especially in robotics, spacecraft and motor control.

In order to realize the sliding mode variable structure control algorithm, there is also need to do a lot of work. Although some algorithm is very perfect in theory, there are still difficult problems in the application, which are that the feedback of the quantities involved whether can been measured or estimated, computing time whether can meet the requirements of online control and so on. The application of sliding mode variable structure control research is still in exploring stage, it is urgent need to carry out the system of practical application.

\section{Acknowledgements}

At the point of finishing this thesis, I'd like to express my sincere thanks to all those who have lent me hands in the course of my writing this thesis.

\section{References}

[1] Dazhong Zheng. Linear System Theory [M]. Beijing: Ttsinghua University Press, (2002)

[2] Yueming Hu. Variable Structure Control Theory and Application [M]. Beijing: Science Press, (2003)

[3] Jinkun Liu. MATLAB Simulation of Sliding Mode Variable Structure Control [M]. Beijing: Ttsinghua University Press, (2005)

[4] Xiaofeng Wei, Changsheng Jiang. Variable Structure Control of Inverted Pendulum Based on State Observer [J]. Journal of Yancheng Institute of Technology (Natural Science Edition), 18 (2005), p. $22-24$

[5] Weiwei Sun, Yuqiang Wu. Fuzzy Sliding Mode Control Algorithm Simulation on a Kind of Multi-joint Robot System [J]. Journal of System Simulation, 17 (2005), p. 1449-1452

[6] Min Tan, Shuo Wang. Research Progress on Robotics [J]. ACTA AUTOMATICA SINICA, 39(2013), p.963-972 